\title{
Hierarchical flower-like ZnO-Ag@Cellulose composite with antifouling and antibacterial properties for efficient recovery of tellurium (IV) from wastewater
}

\section{Guanglei Yao \\ Jiangsu University \\ Xue Shao \\ Jiangsu University \\ Zhiwei Qiu \\ Jiangsu University}

Fengixan Qiu

Jiangsu University

Tao Zhang ( $\sim$ zhangtaochem@163.com )

Jiangsu University https://orcid.org/0000-0001-9255-9802

\section{Research Article}

Keywords: Tellurium, Adsorption, Lignocellulose, Antifouling, Antibacterial

Posted Date: February 12th, 2021

DOI: https://doi.org/10.21203/rs.3.rs-217911/v1

License: (c) (i) This work is licensed under a Creative Commons Attribution 4.0 International License.

Read Full License 


\section{Abstract}

Recovery of the tellurium from wastewater is significance for both industrial applications and sustainable development due to the adverse impacts emanating from environmental pollution and strategic values in the photovoltaic industry. Herein, this study presents the recovery of tellurium from wastewater by flowerlike structure ZnO-Ag@cellulose (ZAC) composites. For adsorbent preparation, lignocellulose was first extracted from waste wood debris by an alkaline extraction process. Then, the flower-like structure of ZAC composites were synthesized by a facile one-step hydrothermal method. The obtained materials are conducted under different parameters to investigate tellurium adsorption properties. The results indicate that the tellurium adsorption process is an exothermic reaction and fits well to the Freundlich model. Kinetic data are well described by pseudo-second-order model, indicating ZAC adsorption of tellurium is a chemical process. Besides, as-prepared ZAC exhibited excellent performance for tellurium elimination from wastewater with a maximum removal efficiency of $98.86 \%$. The ZAC also shows outstanding antifouling performance in polluted environment and can still achieve the rate of $94.23 \%$ for tellurium. Moreover, the ZAC composite reveals high reusability and stability after six desorption-regeneration cycles. Importantly, the ZAC composite exhibits disinfection performance against E. coli and S. aureus with high killing efficiency. Therefore, this work not only demonstrates ZAC composite is efficient adsorbent with antifouling and antibacterial capability for tellurium recovery, but also provides an effective channel for promising candidates to decontaminate similar pollutants and practical applications of wastewater.

\section{Highlights}

1. The ZAC composites were fabricated via a facile one-step hydrothermal method.

2. The raw materials are extracted from waste wood debris using alkaline extraction process to extract lignocellulose.

3. The prepared composite material has high antifouling and antibacterial ability for the adsorption of tellurium (IV).

4. The resulting composite material exhibits excellent performance, with a maximum removal rate of tellurium (IV) was $98.86 \%$.

5. The prepared adsorbent has excellent stability and reusable ability to remove tellurium in wastewater.

\section{Introduction}

Tellurium, as the strategic resource, has become major concern to the public due to its high commercial and industrial value (Liu et al., 2021; Mal et al., 2017). Simultaneously, tellurium as a p-type semiconductor has remarkable thermal conductivity, and exhibits very broad industrial application prospect, especially in photovoltaic (Curtin et al., 2020), metallurgy (Liu et al., 2020), electrical (Chen et al., 2020), glass (Machado and Pereira da Silva, 2020), and ceramic industry. However, the extensive use of 
tellurium-containing materials has engendered a large amount of toxic tellurium-containing wastewater, resulting in serious environmental pollution and destruction of the ecological balance, even it difficult to deal with its long-term effects (Missen et al., 2020; Xu et al., 2020a). With the increase in environmental awareness, tellurium-containing wastewater is an extensive attention problem in industrial development. For instance, arbitrary discharge may cause tellurium-containing wastewater to enter the groundwater system and accumulate in the food chain, making it difficult to biodegrade (Bullock et al., 2018). Additionally, metal smelting and coal burning have caused tellurium (IV) content in industrial wastewater and groundwater to exceed prescribed standards and have a serious threat to human life (Xu et al., 2020b). Therefore, recovery of tellurium from wastewater is of great importance for both industrial applications and sustainable development due to the adverse impacts emanating from environmental pollution and strategic values in photovoltaic industry.

Currently, several separation purification methods have been proposed for the treatment of tellurium from wastewater, such as ion exchange, solvent extraction (Narimani-Sabegh and Noroozian, 2019), membrane separation (Yu et al., 2019), adsorption (Qiu et al., 2020b), and biological treatment processes (Ramos-Ruiz et al., 2016). Among above method, adsorption technology in removal of tellurium from wastewater exhibits many advantages, including high efficiency, simple operation, versatility and environmental friendliness. Thence, variety of nanomaterials, $\mathrm{TiO}_{2}, \mathrm{ZrO}_{2}, \mathrm{NZVFe}$ and $\mathrm{MnO}_{2}$, have been prepared and adopted to remove tellurium in wastewater, which have high adsorption activities and abundant surface functional groups towards the adsorption tellurium processes (Parashar et al., 2017; Wan et al., 2020). For instance, Wu et al. fabricated porous 3D zirconia microspheres, as an efficient adsorbent for tellurium removal (Wu et al., 2019). NZVFe was utilized to adsorb tellurium and exhibit outstanding adsorption capacity (Yu et al., 2018). However, the aggregation problem of powder materials limits their reusability and removal efficiency in aqueous solutions, which also hinders their practical application and further development in adsorption. To overcome the drawbacks of aggregation, Yu et al. presented strategies in powder materials aggregation problem by using CNTs as substrate, synthesized $\mathrm{NZVI/ACNTs} \mathrm{showed} \mathrm{excellent} \mathrm{promising} \mathrm{in} \mathrm{recovery} \mathrm{of} \mathrm{tellurium} \mathrm{(Yu} \mathrm{et} \mathrm{al.,} \mathrm{2019).} \mathrm{But} \mathrm{its} \mathrm{still} \mathrm{suffer} \mathrm{from}$ some issues such as poor stability, complex preparation process, environmental issue, poor antifouling and antibacterial capability. Therefore, the adsorption performance of composites is still a challenge, and it is imperative to develop environmentally friendly, efficient and antibacterial adsorbents to eliminate tellurium.

Recently, ZnO has excellent performance in purifying organic and inorganic pollutants from wastewater, such as removing arsenic (Sharma et al., 2019b), chromium (Sharma et al., 2019a) and dyes (Phan et al., 2020). These behaviors may be derived from the high adsorption efficiency, antibacterial ability (Bhutiya et al., 2018; Zhao et al., 2018), and chemical stability of ZnO making it exhibit fantastic adsorption capacity when eliminating various contaminations from wastewater. But it is worth noting that the $\mathrm{ZnO}$ is also a powder material, which is difficult to actual production application in wastewater. Meanwhile, support materials have been evaluated as significant composition that can avoid aggregation and improve the dispersion of materials (Yang et al., 2021). Cellulose as a renewable support material with 
outstanding performance in wastewater treatment has attracted vast attention on account of its distinct physical, chemical properties and being environmentally friendly (Peng et al., 2016; Wang et al., 2020b). Additionally, as one of the most abundant substances in the world, cellulose has non-toxic, chemical compatibility, low density, superior mechanical properties and high aspect ratio (Anu et al., 2020; Tang et al., 2020; Zhao et al., 2017). To enhance the surface properties of support materials, many researchers have attempted to develop the cellulose composite with relatively low cost and high adsorption capacity. For instance, Zhang and coworkers prepared a layered mesoporous nano- $\mathrm{TiO}_{2} /$ cellulose composite material by microwave-assisted method for rapid adsorption of $\mathrm{Pb}^{2+}$ in wastewater (Zhang et al., 2017), but the application of composite material has been restricted by the inherent interaction of cellulose with proteins, bacteria, and other foulants, which seriously damage the adsorbent and affect the ability of the adsorbent (Fakhre and Ibrahim, 2018). Based on the above defects, Phan et al. successful fabricated $\mathrm{ZnO}-\mathrm{HT}$-PAN, indicating that the $\mathrm{ZnO}$ nanoparticles attached to nanofibers can improve the antibacterial ability (Phan et al., 2020). However, the antibacterial ability of only $\mathrm{ZnO}$ is limited in practical applications. Thence, Huang et al. synthesized Ag@ZnO-OAc NPs, the Ag@ZnO nanoparticles into the membranes to enhance the antibacterial performance of composite (Huang et al., 2020). Moreover, Dumée et al. prepared a bio-antifouling membrane material, employing a novel cold spray technology to embed silver nanoparticles in the membrane to reduce biological pollution and bacteria influence, to achieve the purpose of purifying wastewater, indicating that silver nanoparticles have great prospects in biological antifouling and antibacterial properties (Dumée et al., 2015). To our best knowledge, there was little research on ZnO-Ag composite with antifouling and antibacterial capabilities for adsorption tellurium in wastewater. In addition, understanding of interface characteristics between nanomaterials and cellulose clearly is essential to enhance the adsorption properties.

In this work, a cost-effective and eco-friendly composite is prepared by a facile one-step hydrothermal method. To obtain a high-efficiency adsorbent for tellurium with antifouling and antibacterial capabilities, $\mathrm{ZnO}$ and $\mathrm{Ag}$ are introduced into the surface of cellulose as key substances for treating wastewater. Furthermore, ZAC has more hydroxyl groups on the surface, which is beneficial to achieve the purpose of adsorbing tellurium. This study also discussed the kinetics, interfering ions and thermodynamics in the tellurium adsorption process, in order to have a deeper understanding of the adsorption mechanism, thermodynamics and kinetics. Importantly, the antifouling and antibacterial experiments of ZAC were determined by bovine serum albumin, E. coli and S. aureus, respectively. This work provides a novel orientation for adsorbing tellurium with antifouling and antibacterial capabilities from wastewater, not only for the valorization of waste cellulose resources, but also for large-scale elimination of toxic ion in industrial wastewater.

\section{Materials And Methods}

\subsection{Chemicals and materials}


Sodium tellurite $\left(\mathrm{Na}_{2} \mathrm{TeO}_{3}, \geq 98.0 \%\right)$, Silver nitrate $\left(\mathrm{AgNO}_{3}, \geq 99.8 \%\right)$, ammonia $\left(\mathrm{NH}_{3} \cdot \mathrm{H}_{2} \mathrm{O}, 25.0-28.0 \%\right)$, hydrochloric acid $(\mathrm{HCl}, 36.0-38.0 \%)$, sodium hydroxide $(\mathrm{NaOH}, \geq 97.0 \%)$, sodium nitrate $\left(\mathrm{NaNO}_{3}, \geq 99.0 \%\right)$, sodium chloride $(\mathrm{NaCl}, \geq 99.8 \%)$, disodium hydrogen phosphate $\left(\mathrm{Na}_{2} \mathrm{HPO}_{4} \cdot 12 \mathrm{H}_{2} \mathrm{O}, \geq 99.0 \%\right)$, sodium sulfate $\left(\mathrm{Na}_{2} \mathrm{SO}_{4}, \geq 99.0 \%\right)$, sodium chlorite $\left(\mathrm{NaClO}_{2}, 80.0 \%\right)$ and anhydrous ethanol $\left(\mathrm{CH}_{3} \mathrm{CH}_{2} \mathrm{OH}, 75.0 \%\right)$ were purchased from Sinopharm Chemical Reagent Co., Ltd. China Shanghai. All chemicals were of analytical grade and used without further purification.

\subsection{Lignocellulose extraction}

Lignocellulose was extracted from waste wood debris by an alkaline extraction process. Typically, $30 \mathrm{~g}$ of wood debris was washed several times with deionized water, ethanol, and then dried in an oven at $80^{\circ} \mathrm{C}$ overnight. Then, $15 \mathrm{~g}$ of pre-treated wood debris was dispersed in $100 \mathrm{~mL}$ of $5 \%$ sodium hydroxide solution and stirred at $80^{\circ} \mathrm{C}$ for $6 \mathrm{~h}$ to remove lignin and hemicellulose of wood debris. The suspension was further filtered and washed through deionized water until the $\mathrm{pH}$ reached neutral. Subsequently, the crude cellulose was bleached in a $5 \mathrm{wt} \%(\mathrm{pH}=4)$ sodium chlorite under magnetic stirring at $80^{\circ} \mathrm{C}$ for $6 \mathrm{~h}$. Finally, the lignocellulose was obtained by filtration and washed with water several times, and dried at 80 ${ }^{\circ} \mathrm{C}$ for $6 \mathrm{~h}$.

\subsection{Preparation of ZAC composites}

The ZAC with flower-like structures was prepared by a facile one-step hydrothermal method. In a typical experiment, $0.29 \mathrm{~g}$ zinc nitrate hexahydrate, $5.5 \mathrm{~g}$ cellulose and $0.167 \mathrm{~g}$ silver nitrate were dissolved in 85 $\mathrm{mL}$ of deionized water, and magnetic stirring for $2 \mathrm{~h}$ at room temperature. Then, the reaction system was sonicated for $30 \mathrm{~min}$ at a frequency of $60 \mathrm{kHz}$. After that, a certain amount of ammonia water was slowly dropped into the above mixed solution at a rate of $10 \mathrm{drops} / \mathrm{min}$ until the $\mathrm{pH}$ of the solution was 9 , and transferred to a Teflon stainless steel autoclave for hydrothermal synthesis at $130^{\circ} \mathrm{C}$ for $8 \mathrm{~h}$. Finally, the obtained product was collected by centrifugation and filtration, washed several times with distilled water and absolute ethanol, then dried at $70^{\circ} \mathrm{C}$ for $15 \mathrm{~h}$ under vacuum condition and sealed. The preparation process of ZAC was illustrated in Scheme 1.

Scheme 1. Schematic diagram of the manufacture of ZAC composite materials.

\subsection{Characterization}

The morphology of ZAC was investigated by scanning electron microscopy (SEM FEI, Quanta 200, USA), and the content of elements was measured by energy-dispersive X-ray spectroscopy (EDS, JEM-2100). The transmission electron microscope (TEM) measurement was performed on a JEM-1400 Plus microscope (JEOL Ltd., Japan) for ZAC size with an acceleration voltage of $80 \mathrm{kV}$. The crystal structures of ZAC hybrid nanoparticles were analyzed by X-ray diffraction (XRD, JEOL JDX-3530, Tokyo, Japan) with 
Cu Ka radiation with a scanning speed of $4^{\circ} / \mathrm{min}$ in the $2 \theta$ range from $10^{\circ}$ to $80^{\circ}$. The functional groups on the surface of the sample were analyzed by Fourier Transform Infrared (FT-IR) spectrophotometer (Thermo Nicolet, NEXUS, TM) in the range of 400-4000 $\mathrm{cm}^{-1}$. The surface element composition and chemical valences of ZAC before and after tellurium adsorption were analyzed by X-ray photoelectron spectroscopy (XPS) on Thermo ESCALAB 250 XI. Inductively coupled plasma optical emission spectrometry (ICP-OES, Perkin-Elmer Optima 2100DV) was used to measure the tellurium content in the solution after adsorption.

\subsection{Batch adsorption experiment}

To investigate tellurium adsorption performance of ZAC composites, the batch experiments were conducted under different conditions. For tellurium adsorption, $1 \mathrm{~g} / \mathrm{L}$ tellurium solution was prepared as a stock solution for different concentrations of adsorption experiments. In the batch experiment, the thermodynamic and kinetic, adsorbent dosage, $\mathrm{pH}$ and interfering ions were also investigated. Moreover, the experiments were carried out under the conditions: adsorption dose of $30 \mathrm{mg}$, $\mathrm{pH}$ adjusted with 0.1 $\mathrm{mol} / \mathrm{L}$ sodium hydroxide and hydrochloric acid, temperature of $25^{\circ} \mathrm{C}$ and the adsorption time was $6 \mathrm{~h}$. After adsorption under certain conditions, centrifuge at 10,000 rpm for $15 \mathrm{~min}$ to separate the solid/liquid phase. Finally, the supernatant was filtered, and the concentration of tellurium in the solution was measured by ICP-OES.

Adsorption performance of ZAC for tellurium was evaluated as removal efficiency (R) and adsorption capacity $\left(Q_{t}\right)$ using eqs $1-2$, respectively.

$$
\begin{aligned}
& R(\%)=\frac{\mathrm{C}_{\mathrm{i}}-\mathrm{C}_{\mathrm{e}}}{\mathrm{C}_{\mathrm{e}}^{\prime}} \times 100 \% \\
& \mathrm{Q}_{\mathrm{t}}=\frac{\left(\mathrm{C}_{\mathrm{i}}-\mathrm{C}_{\mathrm{e}}\right) \mathrm{V}}{{ }_{\mathrm{m}}}
\end{aligned}
$$

Where $\mathrm{C}_{\mathrm{i}}$ and $\mathrm{C}_{\mathrm{e}}$ represent the initial concentration and equilibrium concentration of $\mathrm{TeO}_{3}{ }^{2-}$ in solution $(\mathrm{mg} / \mathrm{L})$, respectively, $\mathrm{V}$ is the volume of solution $(\mathrm{mL})$, and $\mathrm{m}$ is the mass of adsorbent $(\mathrm{mg})$.

\subsection{Antifouling experiment}

During the antifouling experiment, bovine serum albumin (BSA) solution was used to simulate the protein pollutions on the surface of the adsorbent. In a typical procedure, BSA $(5.0 \mathrm{~g})$ was dissolved in $50 \mathrm{~mL}$ of Phosphate Buffered Saline (PBS). The ZAC was added to BSA/PBS solution and soaked for $24 \mathrm{~h}$ to make it well contaminated. After that, the adsorbent was washed with distilled water and then used for the tellurium adsorption experiment. 


\subsection{Antibacterial activity tests}

Two classic bacteria Escherichia coli (E. coli) and Staphylococcus aureus (S. aureus) were as the representative model microorganisms to investigate the antibacterial performance of ZAC by viable plate counting technique. The E. coli and S. aureus were cultured in a biochemical incubator at $37^{\circ} \mathrm{C}$ for $24 \mathrm{~h}$, the concentrations of $\mathrm{E}$. coli and S. aureus were adjusted to $10^{8} \mathrm{CFU} / \mathrm{mL}$ with the PBS solution and transferred to agar culture medium. Subsequently, the ZAC $(20 \mathrm{mg})$ mixed with $10 \mathrm{~mL}$ of deionized and ultrasound for $30 \mathrm{~min}$, and then take $1 \mathrm{~mL}$ of the above solution add to the agar medium. Finally, the above mixture was placed in agar medium at biochemical incubator for $24 \mathrm{~h}$. The growth status of bacteria was observed and counted the number of surviving bacteria to explore the antibacterial performance of the samples.

\section{Results And Discussion}

\subsection{Structure and morphology}

The microstructure and surface morphology of ZC and ZAC were determined by SEM. From Fig. 1A, it can be clearly seen that the flower-like structure of zinc oxide has an irregular morphology on the cellulose surface. Meanwhile, the inset of Fig. 1A shows that the flower-like microstructure about $6.0 \mu \mathrm{m}$ in diameter. Besides, the mapping images confirmed that $C, O$ and $Z n$ were successfully loaded, indicating the ZC was successfully prepared. Compared with the ZC, when the silver nanoparticles are successfully deposited on the fiber surface, ZAC exhibits fiber morphology with coarse surface (Fig. 1B). The presence of silver will be increased the roughness (the inset in Fig. B) and antibacterial capability for ZAC, which was consistent with previous research results (Huang et al., 2020). Additionally, the mapping research demonstrates that $C, O, Z n$, and $A g$ were evenly dispersed on the surface of the biomass fiber, suggesting that the composite of ZAC was synthesized. After adsorption of tellurium, the morphology of ZAC changed significantly from rough structure to smooth plane structure and the gap of the particles are filled (Seen inFig. S1), which maybe attributed to successful adsorption of tellurium from the wastewater.

To further illustrate the composition, EDS was used to analyze the element distribution onto ZAC. As shown in Fig. 1C, the EDS research reveals that four elements $(\mathrm{C}, \mathrm{O}, \mathrm{ZnO}$ and $\mathrm{Ag})$ are uniformly distributed in the flower-like structure. The $\mathrm{C}$ atomic content is calculated to be about $38.25 \mathrm{at} \%$. Ag element is also calculated with the content of 5.92 at\%. In addition, the content of $O$ and $Z n$ elements in the composites reached 33.54 and 22.29 at\%, respectively, implying that the surface of ZAC is enrichment functional group, which can be conducive to adsorb tellurium from wastewater. Furthermore, after adsorption of tellurium (Seen in Fig. S2.), the EDS results indicated that the presence of elemental Te in ZAC surface, which confirmed that the adsorption of tellurium on ZAC surface.

Moreover, the ZC and ZAC crystal structures were investigated by XRD and the results are shown in Fig. 1D. Fig. 1D(a) shows that the characteristic peak located at $22.5^{\circ}(200)$ corresponding to the samples of cellulose reported in previous studies, the intensity indicates that the cellulose is relatively pure and no 
impurity peaks (French, 2014; Tang et al., 2020). As shown in Fig. 1D(b), the typical characteristic diffraction peaks at $31.79^{\circ}, 34.44^{\circ}, 36.34^{\circ}, 47.72^{\circ}, 56.72^{\circ}, 63.01^{\circ}, 66.56^{\circ}, 68.09^{\circ}, 69.31^{\circ}, 72.80^{\circ}$ and $77.24^{\circ}$, which are consistent with the JCPDS Card No.36-1451, this reveals that ZC was obtained (Kitsou et al., 2019; R et al., 2018). Meanwhile, compared with ZC, Fig. 1D(c) exhibits that three new peaks at about $38.51^{\circ}, 44.49^{\circ}$ and $64.60^{\circ}$ assigned to the (111), (200) and (220) planes of Ag (JCPDS Card No.040783), indicating the ZAC was fabricated (Wei et al., 2018). Importantly, as shown in Fig. S3, the peak intensity of ZAC has changed significantly after tellurium adsorption, which probably due to the presence of tellurium masks its strength.

The microstructure of the as-prepared ZC and ZAC composites were also characterized by TEM. As shown in Fig. 2A, the images of $\mathrm{ZC}$ showed that the flower-like $\mathrm{ZnO}$ was located on cellulose surface. The magnified image of ZC (Fig. 2B) was obtained with irregular dimension and calculated the size ranges from 1-3 $\mu \mathrm{m}$. Furthermore, Fig. $\mathbf{2 C}$ shows that $\mathrm{ZnO}$ is well dispersed anchored on the cellulose without agglomeration. Compared to ZC邓the morphology (Fig. 2D) of ZAC was no different after modification with Ag, suggesting that the introduced Ag will not damage and affect the flower-like structure stability (Abdel-Karim et al., 2021). In Fig. 2D and F, the ZAC composite shows different sizes of flower-like structures, this structure will facilitate the application of ZAC in the treatment of tellurium from wastewater.

\subsection{Adsorption mechanism}

The adsorption mechanism can better explain the interaction between the adsorbent and the target of tellurium. Thence, the FT-IR spectrum of the ZAC composite material before and after adsorbing tellurium was analyzed, as shown in Fig. 3A.Fig. $3 A$ (a) shows the peak at about $500 \mathrm{~cm}^{-1}$ corresponds to the bonds of $\mathrm{Zn}-\mathrm{O}$ and $\mathrm{Ag}-\mathrm{O}$, indicating that the ZAC composite material was successfully prepared ( $\mathrm{R}$ et al., 2018; Zare et al., 2019). Moreover, the peaks at $1350 \mathrm{~cm}^{-1}$ are attributed to the bending vibration of $\mathrm{Zn}-\mathrm{OH}$, the peak located at $3370 \mathrm{~cm}^{-1}$ is consistent with the stretching vibration of $-\mathrm{OH}$ on the surface of ZAC (Wang et al., 2019). This result indicates that the surface of ZAC is rich in hydroxyl group, which may contribute to enhance the tellurium adsorption capacity. Compared with the spectrum in Fig. 3A(a), Fig. 3A(b) shows the FT-IR spectrum has significantly changed with a new peak appear near $480 \mathrm{~cm}^{-1}$, which is attributed to the vibration of the Te-O bond, confirming that the tellurium was successfully adsorbed on the surface of ZAC (Qiu et al., 2020b). Most importantly, the peak of hydroxyl group is decreased significantly after adsorption of tellurium, revealing that the hydroxyl group on the surface of ZAC participated in the adsorption of tellurium. Therefore, the possible mechanism for ZAC to adsorb tellurium is by ligand exchange between the hydroxyl groups and tellurium ions. Furthermore, the adsorption mechanism can be further confirmed by XPS.

To further better investigated the adsorption mechanism, chemical composition and valence state of the ZAC composites before and after adsorption tellurium, the ZAC composites was carried out by XPS.Fig. 3B(a) reveals the XPS full survey spectrum of 01s, Zn2p, and Ag3d about ZAC composite material before 
adsorption tellurium, indicating the required composite material was prepared. Compared with the virgin ZAC, Te3d peak appeared (Fig. 3B(b)) at binding energy of $576.18 \mathrm{eV}$ and $586.25 \mathrm{eV}$ after adsorption tellurium, these peaks demonstrate that ZAC can adsorb tellurium from wastewater (Yue et al., 2019). In addition, the spectrum of 01s was divided into two peaks at binding energy of $530.55 \mathrm{eV}$ and $532.61 \mathrm{eV}$ (Fig. $3 C(a))$, which were ascribed to the oxygen bonded to metal $(\mathrm{Zn}-0)$ and hydroxyl bonded to metal ( $\mathrm{Zn}-\mathrm{OH})$ (R et al., 2018), respectively. This result shows that the surface of $\mathrm{ZAC}$ is rich in $-\mathrm{OH}$, which is consistent with infrared spectroscopy analysis results. It is worth noting that the peak areas have changed significantly before and after adsorption tellurium, the peak area ratio of $\mathrm{Zn}-\mathrm{O}$ increased (Fig. $3 \mathrm{C}(\mathrm{b})$ ), while the peak area ratio of $\mathrm{Zn}-\mathrm{OH}$ decreased significantly, the reasonable reason may be due to the formation of new bonds such as Te-O bonds, suggesting that the -OH groups are involved in the adsorption process of tellurium. It means that the adsorption of tellurium by ZAC may be attributed to the ion exchange between $\mathrm{TeO}_{3}{ }^{2-}$ and $-\mathrm{OH}$, which is in agreement with the result of FT-IR. From Fig. $3 \mathrm{D}(\mathrm{a})$, the spectrum of Zn2p before adsorption tellurium, the binding energies of $1044.72 \mathrm{eV}$ and $1021.39 \mathrm{eV}$ are assigned to the $Z n 2 p_{1 / 2}$ and $Z n 2 p_{3 / 2}$, respectively (Wang et al., 2020a). The difference between the two binding energies is $23 \mathrm{eV}$, indicating that it is the standard reference value of $\mathrm{ZnO}$ (He et al., 2019). Besides, the peak area ratio of Zn2p decreased (Fig. 3D(b)), further confirmed the Zn2p participated in the tellurium adsorption process. In Fig. 3E(a), the two peaks of binding energies at $367.61 \mathrm{eV}$ and $373.52 \mathrm{eV}$,

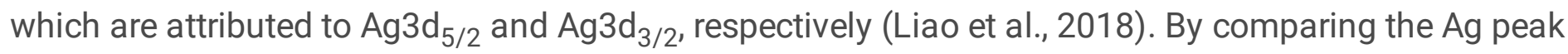
area ratio of ZAC before adsorption tellurium, the Fig. $3 \mathrm{E}(\mathrm{b})$ has a very significant reduction, which may be attributed to Ag formed a chelate with tellurium, making its intensity reduce. Fig. $3 \mathrm{~F}$ shows the spectrum of Te3d after adsorption tellurium, the peaks appeared at $576.18 \mathrm{eV}$ and $586.25 \mathrm{eV}$ corresponded to $T e 3 d_{5 / 2}$ and $T e 3 d_{3 / 2}$, which further demonstrates the successful adsorption of tellurium by ZAC (Weiss et al., 2020). Therefore, the above results are consistent with FT-IR results, confirming that the contaminant tellurium elimination is ascribed to the $-\mathrm{OH}$, which reveals the domination of adsorption mechanism is ion exchange between tellurium (IV) and the $-\mathrm{OH}$ on ZAC. Meanwhile, a schematic illustration of the adsorption process is provided in Scheme 2.

\subsection{Adsorption isotherms and adsorption kinetics}

The equilibrium concentration can well describe the relationship between adsorbent and the concentration of tellurium in wastewater at different temperatures. Hence, the static adsorption experiments of ZAC and ZC at different concentrations (the initial concentrations were $10-100 \mathrm{mg} / \mathrm{L}$, the temperature were at $298.15 \mathrm{~K}, 308.15 \mathrm{~K}$ and $333.15 \mathrm{~K}$, respectively) were carried out and fitting results were fitted by Langmuir isotherm and Freundlich isotherm (The form of these models is shown Table S1), the results are shown in Fig. 4. Fig. 4A shows that $Z C$ is better fitted by Freundlich isotherm $\left(R^{2}=0.987\right.$, 0.988 and 0.957$)$ compared to Langmuir isotherm $\left(R^{2}=0.950,0.896\right.$ and 0.841$)$, indicating that the process of adsorbing tellurium is dominated by multilayer adsorption. The removal efficiency of ZC decreases with increasing temperature, suggesting that this adsorption process is an exothermic reaction (Jin et al., 2020). Moreover, compared with the adsorbent of ZC, Fig. 4D shows that the ZAC has 
outstanding adsorption capacity for tellurium and excellent Freundlich linear fitting coefficient $\left(R^{2}=0.966\right.$, 0.969 and 0.979 ), implying that ZAC has superior potential in the adsorption of tellurium, which may be attributed to the formation of a chelate complex to enhance the adsorption performance of ZAC. Another interesting result was also observed that the adsorption capacity of ZAC increases with the temperature decreased (from $333.15 \mathrm{k}$ to $298.15 \mathrm{k}$ ), indicating that the process of ZAC adsorption tellurium is consistent with the result of $\mathrm{ZC}$, which were more suitable for operation at relatively low temperatures and large-scale applications in the future.

To further investigate the performance of tellurium and the rate-limiting step in the adsorption process, the pseudo-first-order and pseudo-second-order were simulated (The kinetic model is shown Table S1). Fig. 4B shows the pseudo-first-order results of $Z C$, which correlation coefficient $\left(R^{2}\right)$ were only 0.817 , 0.878 and 0.782 , respectively. The pseudo-second-order of ZC (Fig. 4C) with a high correlation coefficient $\left(R^{2}=0.994,0.986\right.$ and 0.996$)$ at three different temperatures $(298.15 \mathrm{~K}, 308.15 \mathrm{~K}$ and $333.15 \mathrm{~K})$, illustrating that the interaction of tellurium and adsorption sites is chemical action. Fig. $4 \mathrm{E}$ reveals the pseudo-first-order mode results of ZAC with correlation coefficients of $0.967,0.878$, and 0.782 for 298.15 , 308.15 , and $333.15 \mathrm{~K}$, respectively, which suggest that the process of adsorption tellurium is not proper for the physical adsorption process on the ZAC surface. Furthermore, compare with Fig. 4E, the pseudosecond-order model (Fig. 4F) describes the kinetics of tellurium adsorption onto ZAC gave a higher correlation coefficient $\left(R^{2}=0.997,0.999\right.$ and 0.998$)$, demonstrating that the adsorption of tellurium is mostly governed by chemical adsorption in wastewater.

\subsection{Adsorption studies}

To investigate the surface charge of ZAC, the zeta potential was measured at different pH values, which have a critical impact on the stability of liquid dispersions. As shown in Fig. 5A, the zeta potential of ZAC decreases with the increase of $\mathrm{pH}$, exhibiting that the surface property of ZAC is negative at $\mathrm{pH} 2-11$. In addition, the $\mathrm{pH}$ plays a significant effect on tellurium adsorption performance. Fig. $5 \mathrm{~B}$ shows that the adsorption capacity of ZC and ZAC are strongly dependently on different $\mathrm{pH}$. As shown in the $\mathrm{pH}$ range of $2-6$, it is found that the removal efficiency of $Z C$ increase from $78.12 \%$ to $82.34 \%$ with increasing of $\mathrm{pH}$. However, the tellurium adsorption performance of ZAC attains its maximum removal efficiency of $98.86 \%$, which may be due to the protonation and the functional groups on the ZAC surface, making the availability of ZAC adsorbent sites get more active and increase the interaction of tellurium, resulting in ZAC with excellent tellurium adsorption capacity. Moreover, the adsorption capacity of ZC and ZAC decrease sharply as the $\mathrm{pH}$ increase (between $\mathrm{pH} 7-11$ ), this phenomenon will attribute to the deprotonation and the surface of ZAC be dominated by negative charge. Meanwhile, it should be noted that tellurium mainly exists in the form of $\mathrm{TeO}_{3}{ }^{2-}$ and $\mathrm{HTeO}^{3-}$ under acidic and alkaline conditions, respectively, the zeta potential of ZAC is always negative at $\mathrm{pH} 2-11$, making the repulsive force increase between ZAC and the target of tellurium. Therefore, the results indicate that ZAC has outstanding removal efficiency for tellurium under weak acid environment. 
To evaluate the application selectivity possibility of ZAC in practical wastewater, the presence of some ions $\left(\mathrm{CO}_{3}{ }^{2-}, \mathrm{SO}_{4}{ }^{2-}, \mathrm{Cl}^{-}\right.$and $\left.\mathrm{NO}_{3}{ }^{-}\right)$in tellurium-containing wastewater, which has significant affect on the adsorption efficiency. As shown in Fig. 6A the similar results were found on ZC and ZAC, the presence of the $\mathrm{NO}_{3}{ }^{-}, \mathrm{Cl}^{-}$and $\mathrm{SO}_{4}{ }^{2-}$ had an unnoticeable effect on the tellurium adsorption, suggesting that the ZAC possess a good selectivity for tellurium in complex environment. However, when $\mathrm{CO}_{3}{ }^{2-}$ was present, the removal rate of tellurium by ZAC decreased to $72 \%$, tellurium adsorption on ZAC surface was restrained by $\mathrm{CO}_{3}{ }^{2-}$, which can be attributed to the competition with tellurium for the active sites in ZAC, leading to the poor adsorption capability of ZAC. On the other hand, studies from the effect of pH indicate the alkaline conditions inhibit tellurium adsorption. Another explanation may be associated with the hydrolysis of carbonate, making the solution to be alkaline. This also has been reported by other researchers and has similar suppression effect on the presence of coexisting anions (Qiu et al., 2020a).

The regeneration ability of the adsorbent is crucial for evaluating the industrial application and economics, the adsorbent of ZAC was investigated by adsorption and desorption processes. In this work, the adsorbed tellurium can be eluted from ZAC surface by using $\mathrm{NaOH}$ solution $(0.5 \mathrm{M})$ as the desorption agent. Fig. $6 \mathrm{~B}$ reveals that the regeneration of adsorbent decreased gradually with the cycle time increased. After three regeneration cycles, the removal efficiency maintained $88.6 \%$ and $73.6 \%$ by the adsorbent of ZAC and ZC, respectively, the adsorption efficiency decrease may be due to the active sites is damaged on the ZAC surface. Moreover, the tellurium adsorption of ZAC exhibits excellent performance than previously reported (Narimani-Sabegh and Noroozian, 2019), indicating that the promising application potential of ZAC can be used in the field of wastewater. Meanwhile, the ZAC can still maintain high adsorption capacity in six desorption-regeneration cycles, and the tellurium removal efficiency was still $72.1 \%$, this phenomenon is because of the reduction of available active sites onto ZAC. In addition, another reason was attributed to the low desorption rate with increase cycles, resulting in lower removal capacities for tellurium. The above results show that the performance of ZAC and further confirm that meet the requirement of regeneration ability for adsorption.

Fig. $6 \mathrm{C}$ shows that the effect of adsorbent dosage on tellurium adsorption processes. It is found that the removal efficiency of tellurium (IV) increased from $71.23 \%$ to $83.46 \%$ when ZC dosage increased from 5 to $35 \mathrm{mg}$, and then kept constant at 35 to $50 \mathrm{mg}$, which is due to the ZC surface have more active site with the increase of adsorbent dosage, making more tellurium to be adsorbed on the ZC surface and increasing the removal efficiency. Importantly, compared to ZC dosage, the removal efficiency of ZAC reaches a maximum value of $98.86 \%$, when the adsorbent dosage increased from $5 \mathrm{mg}$ to $30 \mathrm{mg}$ and eventually reaches equilibrium. This reason may be due to the concentration of tellurium is certain, with the increase of adsorbent dosage, the total amount of adsorption active site on the ZAC surface is increased, indicating that the availability of active sites is unsaturated during the adsorption process. However, the adsorbent dosage increased from 30 to $50 \mathrm{mg}$, the removal efficiency of tellurium does not change much, which may be limited by the content of tellurium. More importantly, the ZAC shows excellent removal efficiency for tellurium than $\mathrm{ZC}$ under the whole dosage range, implying that the introduction of Ag onto ZC, which can enhance the adsorption capacity for tellurium. Therefore, the high 
removal efficiency of ZAC, demonstrated that ZAC could be regarded as a promising material for adsorption of tellurium-containing wastewater and purification of sewage.

Fig.6D shows the tellurium removal efficiency of ZC and ZAC at different initial concentrations. The maximum removal efficiency of only $78.80 \%$ is obtained by $Z C$ at concentration of $20 \mathrm{mg} / \mathrm{L}$, indicating that ZC has low removal efficiency on tellurium and is not suitable for adsorption application. However, even if the tellurium concentration rises to $40 \mathrm{mg} / \mathrm{L}$, the removal efficiency of tellurium can still maintain a maximum removal efficiency of $98.86 \%$. The outstanding adsorption efficiency of ZAC for tellurium elimination results from the interaction between $Z A C$ and tellurium, which shows the stronger ion exchange capacity of ZAC. In addition, either ZC or ZAC adsorption tellurium, a similar tendency can be observed at the range of $40-100 \mathrm{mg} / \mathrm{L}$, and the value of tellurium adsorption on ZC and ZAC decreased. When the concentration of tellurium is below $40 \mathrm{mg} / \mathrm{L}$, the removal efficiency of tellurium is dominated by $-\mathrm{OH}$, but when the concentration of tellurium from $40-100 \mathrm{mg} / \mathrm{L}$, the tellurium will play an increasingly dominant role in the adsorption process, this can be well explained that why the removal efficiency of tellurium decrease as the concentration increases, implying that this is a trade-off presence between removal efficiency and the concentration of tellurium during the adsorption process. Therefore, the above data confirms that the ZAC has excellent adsorption efficiency at a certain concentration of tellurium.

\subsection{Stability}

The stability is critical parameter to illustrate the removal efficiency of the prepared composite for tellurium in wastewater. In this study, the leaching concentration of $\mathrm{Zn}$ and Ag were described to evaluate the stability. As depicted in Fig. 7, it was found that the concentrations of Ag reach the maximum value of $0.24 \mathrm{mg} / \mathrm{L}$ and keeps steady for the increase of time, indicating that the outstanding stability of the Ag can strong anchoring of the ZAC surface. In addition, the trend of $\mathrm{Zn}$ and $\mathrm{Ag}$ was similar when the time is below $40 \mathrm{~min}$, but when the time exceeds $40 \mathrm{~min}$, the presence of $\mathrm{Zn}$ in simulation wastewater slight increase, and the maximum concentration value is $0.27 \mathrm{mg} / \mathrm{L}$. This is probably since the $\mathrm{Zn}$ reaction is not complete during preparation process or part of $\mathrm{Zn}$ falls off, which will all increase the concentration of $\mathrm{Zn}$. It was noted that the above concentration of $\mathrm{Zn}$ and $\mathrm{Ag}$ can be negligible due to the low concentration in wastewater, implying that the ZAC possess a good stability during the adsorption process.

\subsection{Antifouling property}

In general, the presence of macromolecules, biological substances, salts and particulate substances in wastewater, seriously affect the adsorption capacity of adsorbent and limit its practical application in industry. In this work, BSA was selected as contamination model due to the protein have strong adhesion capacity on the adsorbent surface. As illustrated in Fig 8D, it is noticeable that the tellurium removal efficiency of ZC maximum is only $77.56 \%$ as the adsorbent dosage from $5-50 \mathrm{mg}$, compared to the uncontaminated ZC (83.46\%), which gets a poor removal efficiency, confirming that the BSA affects the 
adsorption capacity of ZC. However, ZAC still has strong removal efficiency for tellurium and the maximum removal efficiency can still reach $94.23 \%$, indicating that ZAC has excellent antifouling properties. BSA can affect the adsorption capacity of adsorbent by destroying the adsorbent surface. Meanwhile, the obvious difference in antifouling performance between ZC and ZAC may be associated with the disparate surface chemical material. These results suggest that the presence of Ag may have strong hydration capability, which was attributed to the hydroxyl groups possibly created large hydrophilic repulsion force as a barrier between the contaminants and ZAC, contributing to detachment of the pollutants, revealing that the introduction of Ag improve the anti-fouling performance of the material. On the other hand, the BSA zeta potential is -3.88 mV (Abdel-Karim et al., 2021), the zeta potential of ZAC is also negative, which will increase the surface of repulsion between the contaminants and ZAC surface, and obstruct the contaminants adsorbed on the ZAC surface. Therefore, the antifouling results demonstrate clearly that the ZAC exhibits remarkable removal efficiency for tellurium and can be used in practical applications.

\subsection{Antibacterial activity}

The antibacterial performance of ZC and ZAC were investigated by viable plate counting technique against E. coli and S. aureus, which can be applied as an intuitive to examine the antibacterial ability and informed from the survival situation on the culture plate (Sun et al., 2020). As shown in Fig. 9A, for the blank control, the dense bacterial colonies survive on the culture plate as small white dots. Compared to blank control, it can be clearly found from Fig. 9B that the reduction in survival colonies in E. coli, suggesting that ZC has a certain sterilization ability. But in Fig. $9 \mathrm{C}$, the colony number of E. coli decreases dramatically, implying that the as-fabricated of ZAC exhibit a strong bactericidal performance against the same bacterium (E. coli), confirming that the introduction of Ag can improve the ability of antibacterial (Umapathi et al., 2019). Fig. 9D shows that the S. aureus grows well in the blank control. According to Fig. $9 \mathrm{E}$, the slight inhibition effect suggests that ZC has lower biocide ability. Importantly, it is found that few bacterial colonies were survived in Fig. 9F, this result demonstrates that the outstanding antibacterial performance of ZAC is originated from the introduction of Ag on the ZC surface. Therefore, the above results reveal that ZAC not only has excellent removal efficiency for tellurium in wastewater, but also has very strong antibacterial capability against $\mathrm{E}$. coli and $\mathrm{S}$. aureus.

\section{Conclusions}

In this work, the flower-like structure of ZAC composites were successful synthesized by a facile hydrothermal with brilliant adsorption capacity, reusability, antifouling and antibacterial ability for tellurium remove. Compared to ZC, the as-perpetrated ZAC process an extraordinarily excellent performance with a maximum removal efficiency of $98.86 \%$ for tellurium. Antifouling experiment exhibits that ZAC has strong removal efficiency for tellurium and the maximum value can still reach $94.23 \%$. Besides, the ZAC fits well with Freundlich and Pseudo-second-order kinetic model, revealing that the adsorption process is multilayer chemical adsorption process. The coexisting anion and stability 
adsorption data indicate that ZAC has good selectivity and excellent stability for tellurium. Furthermore, the ZAC also exhibits good reusability still with the removal efficiency of $72.1 \%$ after six desorptionregeneration cycles. Importantly, the antibacterial activity demonstrates that the outstanding antibacterial performance of ZAC against $\mathrm{E}$. coli and $\mathrm{S}$. aureus, which are probably attributed to the introduction of $\mathrm{Ag}$ on the ZC surface. Therefore, the above results suggest that ZAC has outstanding adsorption performance, antifouling and antibacterial ability and is regarded as an eco-friendly and promising adsorption material for the treatment of wastewater.

\section{Declarations}

\section{Acknowledgments}

This work was financially supported by the National Natural Science Foundation of China (21706100 and 21878132), Youth Talent Cultivation Program of Jiangsu University, High-Level Personnel Training Project of Jiangsu Province (BRA2016142), Key Laboratory of Functional Molecular Solids Foundation (FMS201907).

\section{Declaration of Interest Statement}

No conflict of interest exits in the submission of this manuscript, and manuscript is approved by all authors for publication. I would like to declare on behalf of my co-authors that the work described was original research that has not been submitted previously.

\section{References}

Abdel-Karim, A., El-Naggar, M.E., Radwan, E.K., Mohamed, I.M., Azaam, M., Kenawy, E.-R., 2021. Highperformance mixed-matrix membranes enabled by organically/inorganic modified montmorillonite for the treatment of hazardous textile wastewater. Chem. Eng. J. 405, 126964.

Anu, Kumar, A., Rapoport, A., Kunze, G., Kumar, S., Singh, D., Singh, B., 2020. Multifarious pretreatment strategies for the lignocellulosic substrates for the generation of renewable and sustainable biofuels: A review. Renewable Energy.

Bhutiya, P.L., Mahajan, M.S., Abdul Rasheed, M., Pandey, M., Zaheer Hasan, S., Misra, N., 2018. Zinc oxide nanorod clusters deposited seaweed cellulose sheet for antimicrobial activity. Int. J. Biol. Macromol. 112, 1264-1271.

Bullock, L.A., Perez, M., Armstrong, J.G., Parnell, J., Still, J., Feldmann, J., 2018. Selenium and tellurium resources in Kisgruva Proterozoic volcanogenic massive sulphide deposit (Norway). Ore Geol. Rev. 99, 411-424.

Chen, X.-Y., Zhang, J., Du, K.-R., Xie, Q., Hou, Z.-L., 2020. Sb2Te3 nanosheets: Topological insulators of extraordinary electromagnetic response behaviors. Chem. Eng. J. 128036. 
Curtin, A.M., Vail, C.A., Buckley, H.L., 2020. CdTe in thin film photovoltaic cells: Interventions to protect drinking water in production and end-of-life. Water-Energy Nexus. 3, 15-28.

Dumée, L.F., He, L., King, P.C., Moing, M.L., Güller, I., Duke, M., Hodgson, P.D., Gray, S., Poole, A.J., Kong, L., 2015. Towards integrated anti-microbial capabilities: Novel bio-fouling resistant membranes by high velocity embedment of silver particles. J. Membr. Sci. 475, 552-561.

Fakhre, N.A., Ibrahim, B.M., 2018. The use of new chemically modified cellulose for heavy metal ion adsorption. J. Hazard. Mater. 343, 324-331.

French, A.D., 2014. Idealized powder diffraction patterns for cellulose polymorphs. Cellulose. 21, 885-896.

He, X., Yang, D.-P., Zhang, X., Liu, M., Kang, Z., Lin, C., Jia, N., Luque, R., 2019. Waste eggshell membranetemplated $\mathrm{CuO}-\mathrm{ZnO}$ nanocomposites with enhanced adsorption, catalysis and antibacterial properties for water purification. Chem. Eng. J. 369, 621-633.

Huang, X., Chen, Y., Feng, X., Hu, X., Zhang, Y., Liu, L., 2020. Incorporation of oleic acid-modified Ag@ZnO core-shell nanoparticles into thin film composite membranes for enhanced antifouling and antibacterial properties. J. Membr. Sci. 602, 117956.

Jin, L.H., Bai, Y., Li, C.S., Feng, J.Q., Lei, L., Zhao, G.Y., Gao, L., Zhang, P.X., 2020. Effect of fluorine on the intermediate phase evolution for GdBCO film growth. Mater. Chem. Phys. 239, 122054.

Kitsou, I., Panagopoulos, P., Maggos, T., Tsetsekou, A., 2019. ZnO-coated SiO2 nanocatalyst preparation and its photocatalytic activity over nitric oxides as an alternative material to pure ZnO. Appl. Surf. Sci. $473,40-48$.

Liao, G., Li, Q., Zhao, W., Pang, Q., Gao, H., Xu, Z., 2018. In-situ construction of novel silver nanoparticle decorated polymeric spheres as highly active and stable catalysts for reduction of methylene blue dye. Applied Catalysis A: General. 549, 102-111.

Liu, W., Jia, R., Sun, B., Zhang, D., Chen, L., Yang, T., Lu, S., 2020. A novel process for extracting tellurium from the calcine of copper anode slime via continuous enrichment. J. Cleaner Prod. 264, 121637.

Liu, Y., Liu, P., Jiang, Q., Jiang, F., Liu, J., Liu, G., Liu, C., Du, Y., Xu, J., 2021. Organic/inorganic hybrid for flexible thermoelectric fibers. Chem. Eng. J. 405, 126510.

Machado, T.M., Pereira da Silva, M.A., 2020. Preparation and properties of antimony sodium polyphosphate glasses containing tellurium particles. Mater. Chem. Phys. 244, 122731.

Mal, J., Nancharaiah, Y.V., Maheshwari, N., van Hullebusch, E.D., Lens, P.N., 2017. Continuous removal and recovery of tellurium in an upflow anaerobic granular sludge bed reactor. J. Hazard. Mater. 327, 79-88. 
Missen, O.P., Ram, R., Mills, S.J., Etschmann, B., Reith, F., Shuster, J., Smith, D.J., Brugger, J., 2020. Love is in the Earth: A review of tellurium (bio)geochemistry in surface environments. Earth Sci. Rev. 204, 103150.

Narimani-Sabegh, S., Noroozian, E., 2019. Magnetic solid-phase extraction with copper ferrite nanoparticles for the separation and preconcentration of ultra-trace amounts of tellurium (IV) ion in aqueous samples. J. Iran. Chem. Soc. 16, 73-81.

Parashar, K., Ballav, N., Debnath, S., Pillay, K., Maity, A., 2017. Hydrous $\mathrm{ZrO}_{2}$ decorated polyaniline nanofibres: Synthesis, characterization and application as an efficient adsorbent for water defluoridation. J. Colloid Interface Sci. 508, 342-358.

Peng, N., Hu, D., Zeng, J., Li, Y., Liang, L., Chang, C., 2016. Superabsorbent Cellulose-Clay Nanocomposite Hydrogels for Highly Efficient Removal of Dye in Water. ACS Sustainable Chemistry \& Engineering. 4, 7217-7224.

Phan, D.-N., Rebia, R.A., Saito, Y., Kharaghani, D., Khatri, M., Tanaka, T., Lee, H., Kim, I.-S., 2020. Zinc oxide nanoparticles attached to polyacrylonitrile nanofibers with hinokitiol as gluing agent for synergistic antibacterial activities and effective dye removal. J. Ind. Eng. Chem. 85, 258-268.

Qiu, Z., Shi, S., Qiu, F., Xu, X., Yang, D., Zhang, T., 2020a. Enhanced As(Ш) removal from aqueous solutions by recyclable Cu@MNM composite membranes via synergistic oxidation and absorption. Water Res. 168, 115147.

Qiu, Z., Wang, M., Zhang, T., Yang, D., Qiu, F., 2020b. In-situ fabrication of dynamic and recyclable TiO2 coated bacterial cellulose membranes as an efficient hybrid absorbent for tellurium extraction. Cellulose. $27,4591-4608$.

R, S.G., M, N., Patil, V.L., S, P., C, M., Kawasaki, S., Patil, P.S., Hayakawa, Y., 2018. Sensitivity enhancement of ammonia gas sensor based on $\mathrm{Ag} / \mathrm{ZnO}$ flower and nanoellipsoids at low temperature. Sens. Actuators, B. $255,672-683$.

Ramos-Ruiz, A., Field, J.A., Wilkening, J.V., Sierra-Alvarez, R., 2016. Recovery of Elemental Tellurium Nanoparticles by the Reduction of Tellurium Oxyanions in a Methanogenic Microbial Consortium. Environ. Sci. Technol. 50, 1492-1500.

Sharma, M., Joshi, M., Nigam, S., Shree, S., Avasthi, D.K., Adelung, R., Srivastava, S.K., Kumar Mishra, Y., 2019a. ZnO tetrapods and activated carbon based hybrid composite: Adsorbents for enhanced decontamination of hexavalent chromium from aqueous solution. Chem. Eng. J. 358, 540-551.

Sharma, P.R., Sharma, S.K., Antoine, R., Hsiao, B.S., 2019b. Efficient Removal of Arsenic Using Zinc Oxide Nanocrystal-Decorated Regenerated Microfibrillated Cellulose Scaffolds. ACS Sustainable Chemistry \& Engineering. 7, 6140-6151. 
Sun, J., Fan, Y., Ye, W., Tian, L., Niu, S., Ming, W., Zhao, J., Ren, L., 2020. Near-infrared light triggered photodynamic and nitric oxide synergistic antibacterial nanocomposite membrane. Chem. Eng. J. 128049.

Tang, N., Li, Y., Ge, J., Si, Y., Yu, J., Yin, X., Ding, B., 2020. Ultrathin Cellulose Voronoi-Nanonet Membranes Enable High-Flux and Energy-Saving Water Purification. ACS Appl. Mater. Interfaces.

Umapathi, A., Nagaraju, N.P., Madhyastha, H., Jain, D., Srinivas, S.P., Rotello, V.M., Daima, H.K., 2019. Highly efficient and selective antimicrobial isonicotinylhydrazide-coated polyoxometalate-functionalized silver nanoparticles. Colloids Surf., B. 184, 110522.

Wan, S., Qiu, L., Li, Y., Sun, J., Gao, B., He, F., Wan, W., 2020. Accelerated antimony and copper removal by manganese oxide embedded in biochar with enlarged pore structure. Chem. Eng. J. 402, 126021.

Wang, F., Wang, K., Muhammad, Y., Wei, Y., Shao, L., Wang, X., 2019. Preparation of $\mathrm{CeO}_{2} @ \mathrm{SiO}_{2}$ Microspheres by a Non-sintering Strategy for Highly Selective and Continuous Adsorption of Fluoride lons from Wastewater. ACS Sustainable Chemistry \& Engineering. 7, 14716-14726.

Wang, S., Jia, F., Wang, X., Hu, L., Sun, Y., Yin, G., Zhou, T., Feng, Z., Kumar, P., Liu, B., 2020a. Fabrication of ZnO Nanoparticles Modified by Uniformly Dispersed Ag Nanoparticles: Enhancement of Gas Sensing Performance. ACS Omega. 5, 5209-5218.

Wang, Z., Xu, J., Pati, S., Chen, T., Deng, Y., Dewangan, N., Meng, L., Lin, J.Y.S., Kawi, S., 2020b. High H2 permeable SAPO-34 hollow fiber membrane for high temperature propane dehydrogenation application. AlChE J. 66, e16278.

Wei, Y., Wang, X., Yi, G., Zhou, L., Cao, J., Sun, G., Chen, Z., Bala, H., Zhang, Z., 2018. Hydrothermal synthesis of Ag modified $\mathrm{ZnO}$ nanorods and their enhanced ethanol-sensing properties. Mater. Sci. Semicond. Process. 75, 327-333.

Weiss, M., Wirth, B., Marschall, R., 2020. Photoinduced Defect and Surface Chemistry of Niobium Tellurium Oxides ANbTeO6 (A = K, Rb, Cs) with Defect-Pyrochlore Structure. Inorg. Chem.

Wu, X., Guo, X., Zhang, L., 2019. Fabrication of Porous Zirconia Microspheres as an Efficient Adsorbent for Removal and Recovery of Trace Se(IV) and Te(IV). Ind. Eng. Chem. Res. 58, 342-349.

Xu, L., Xiong, Y., Song, Y., Zhang, G., Zhang, F., Yang, Y., Hua, Z., Tian, Y., You, J., Zhao, Z., 2020a. Recycling of copper telluride from copper anode slime processing: Toward efficient recovery of tellurium and copper. Hydrometallurgy. 196, 105436.

Xu, L., Xiong, Y., Zhang, G., Zhang, F., Yang, Y., Hua, Z., Tian, Y., You, J., Zhao, Z., 2020b. An environmentalfriendly process for recovery of tellurium and copper from copper telluride. J. Cleaner Prod. 272, 122723. 
Yang, W., Hu, W., Zhang, J., Wang, W., Cai, R., Pan, M., Huang, C., Chen, X., Yan, B., Zeng, H., 2021. Tannic acid/Fe3+ functionalized magnetic graphene oxide nanocomposite with high loading of silver nanoparticles as ultra-efficient catalyst and disinfectant for wastewater treatment. Chem. Eng. J. 405, 126629.

Yu, H., Chu, Y., Zhang, T., Yu, L., Yang, D., Qiu, F., Yuan, D., 2018. Recovery of tellurium from aqueous solutions by adsorption with magnetic nanoscale zero-valent iron (NZVFe). Hydrometallurgy. 177, 1-8.

Yu, H., Zhang, T., Jing, Z., Xu, J., Qiu, F., Yang, D., Yu, L., 2019. In situ fabrication of dynamic nano zerovalent iron/activated carbon nanotubes membranes for tellurium separation. Chem. Eng. Sci. 205, 278286.

Yue, X., Chen, H., Zhang, T., Qiu, Z., Qiu, F., Yang, D., 2019. Controllable fabrication of tendril-inspired hierarchical hybrid membrane for efficient recovering tellurium from photovoltaic waste. J. Cleaner Prod. 230, 966-973.

Zare, M., Namratha, K., llyas, S., Hezam, A., Mathur, S., Byrappa, K., 2019. Smart Fortified PHBV-CS Biopolymer with ZnO-Ag Nanocomposites for Enhanced Shelf Life of Food Packaging. ACS Appl. Mater. Interfaces. 11, 48309-48320.

Zhang, J., Li, L., Li, Y., Yang, C., 2017. Microwave-assisted synthesis of hierarchical mesoporous nanoTiO2/cellulose composites for rapid adsorption of Pb2+. Chem. Eng. J. 313, 1132-1141.

Zhao, S.-W., Guo, C.-R., Hu, Y.-Z., Guo, Y.-R., Pan, Q.-J., 2018. The preparation and antibacterial activity of cellulose/ZnO composite: a review. Open Chemistry. 16, 9-20.

Zhao, S.-W., Zheng, M., Zou, X.-H., Guo, Y., Pan, Q.-J., 2017. Self-Assembly of Hierarchically Structured Cellulose@ZnO Composite in Solid-Liquid Homogeneous Phase: Synthesis, DFT Calculations, and Enhanced Antibacterial Activities. ACS Sustainable Chemistry \& Engineering. 5, 6585-6596.

\section{Figures}



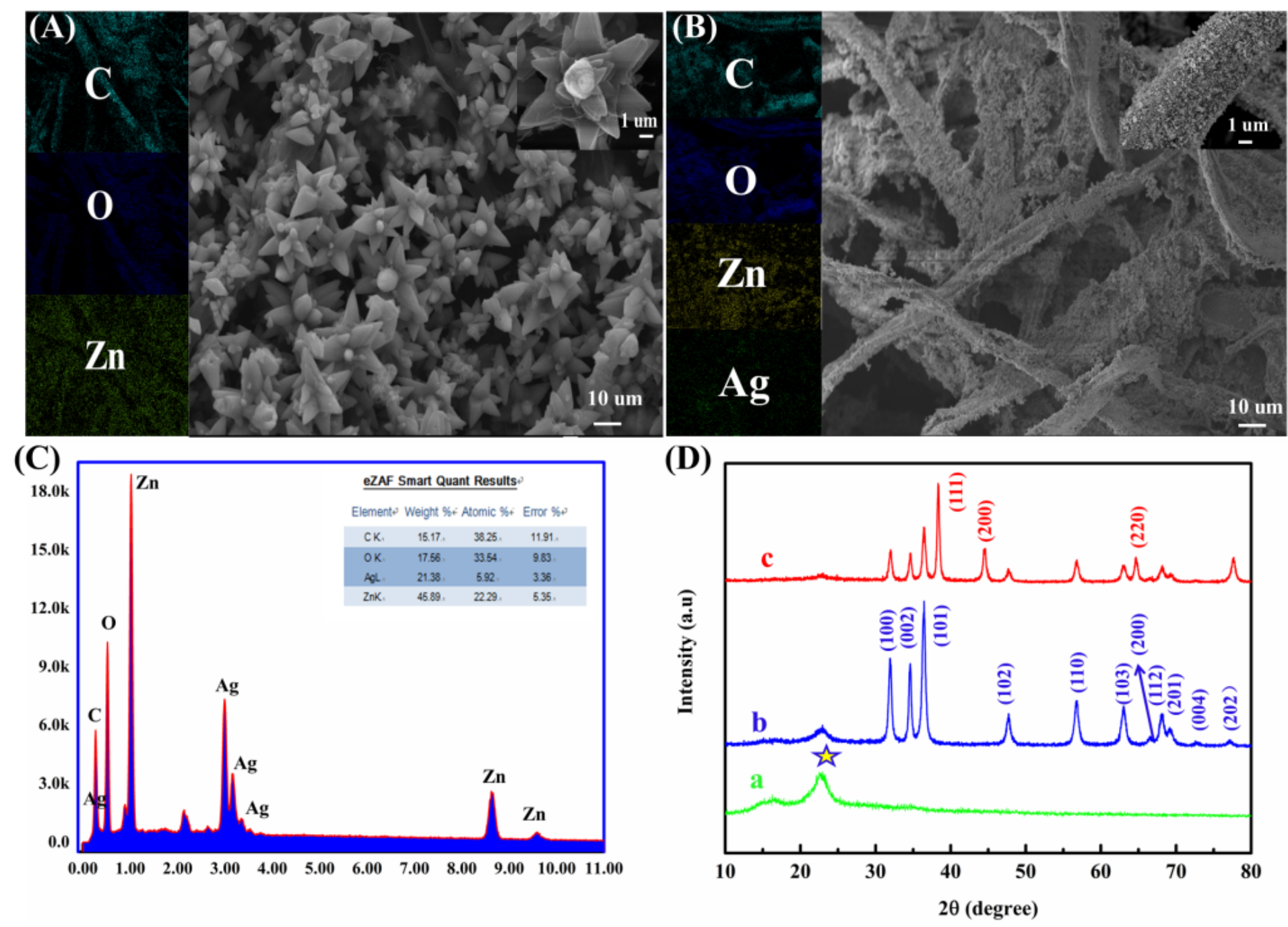

Figure 1

SEM and mapping images of ZC (A); ZAC (B); EDS results of ZAC (C); (D) XRD patterns of: (a) cellulose, (b) ZC, (c) ZAC. 

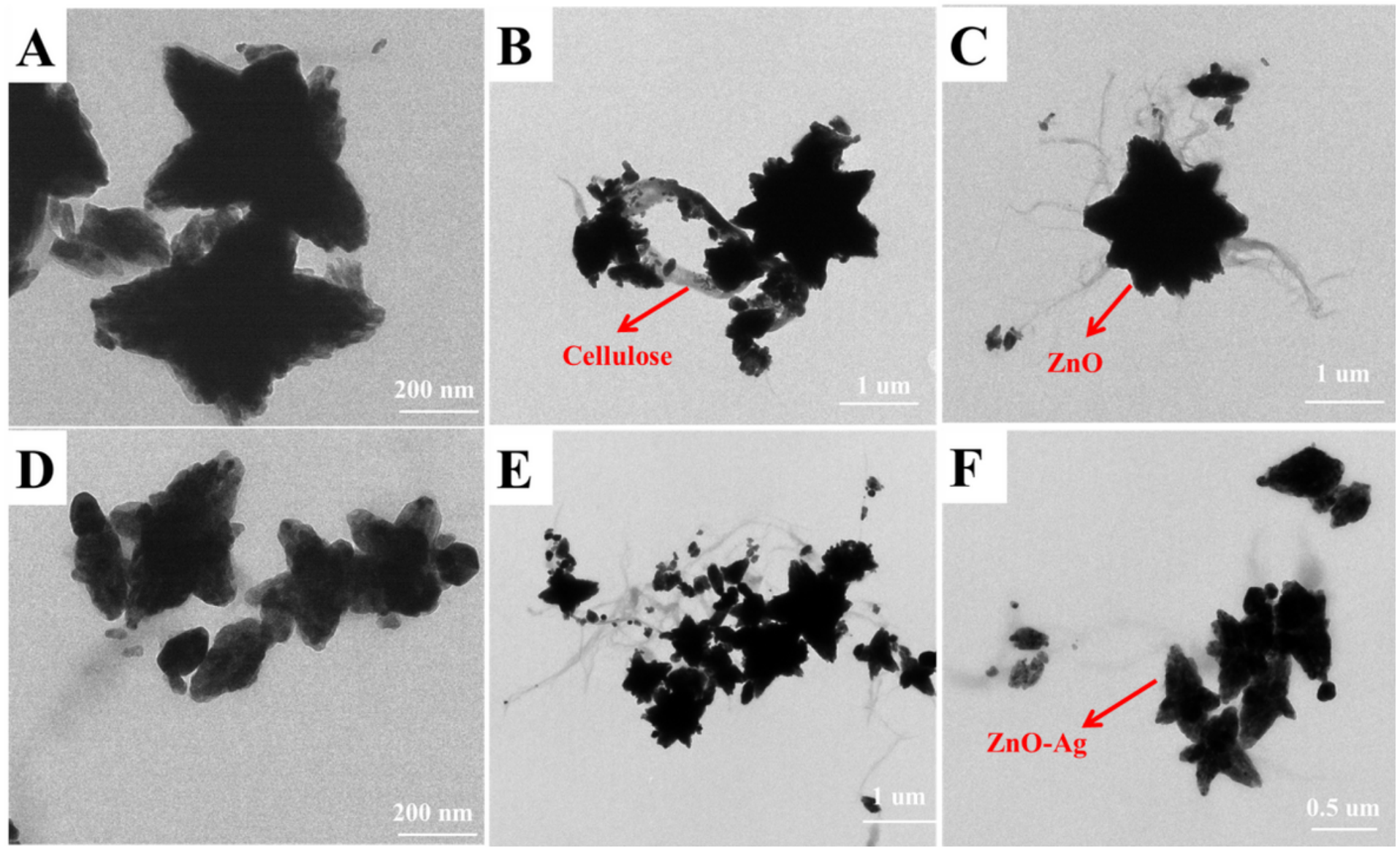

\section{Figure 2}

TEM images of ZC $(A, B, C)$ and $Z A C(D, E, F)$.
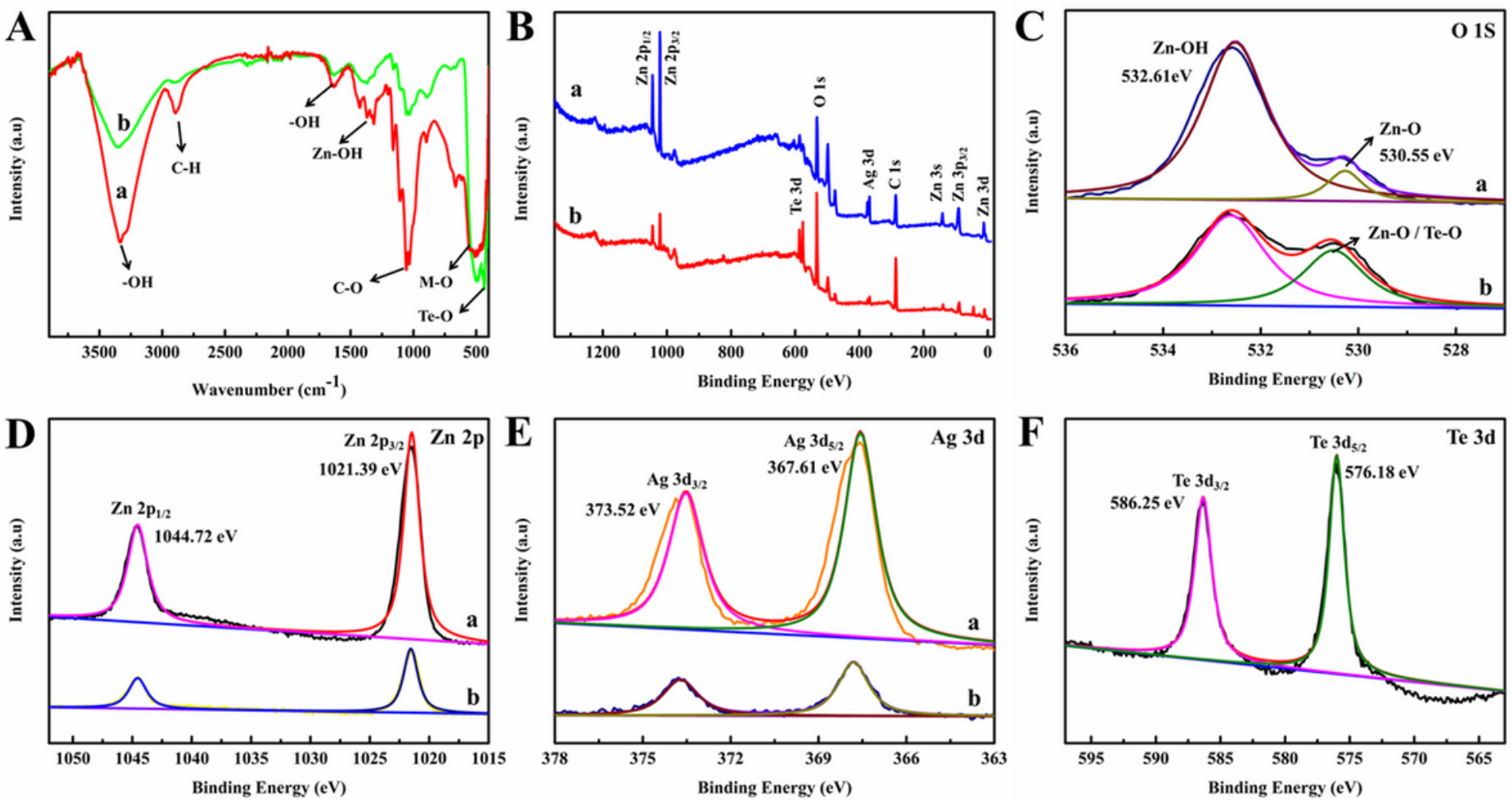
The FT-IR spectra of ZAC composites A; XPS spectra of ZAC before and after adsorption tellurium: (B) ZAC composites, (C) 01s, (D) Zn2p, (E) Ag3d, (F) Te3d (a before adsorption, b after adsorption).
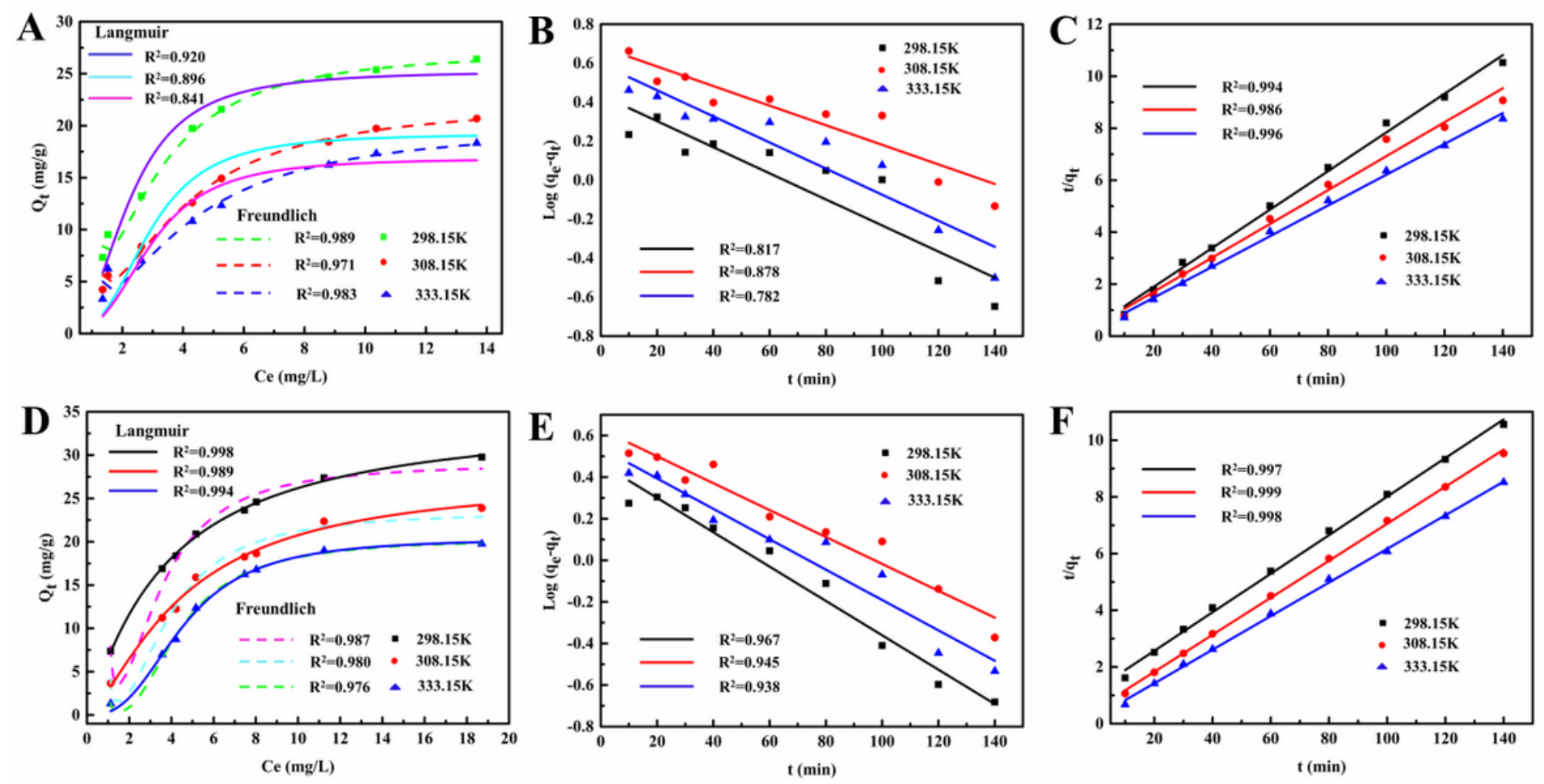

Figure 4

ZC: Langmuir isotherm and Freundlich isotherm (A), Pseudo-first-order mode (B), Pseudo-second-order mode (C). ZAC: Langmuir isotherm and Freundlich isotherm (D), Pseudo-first-order mode (E), Pseudosecond-order mode $(F)$.
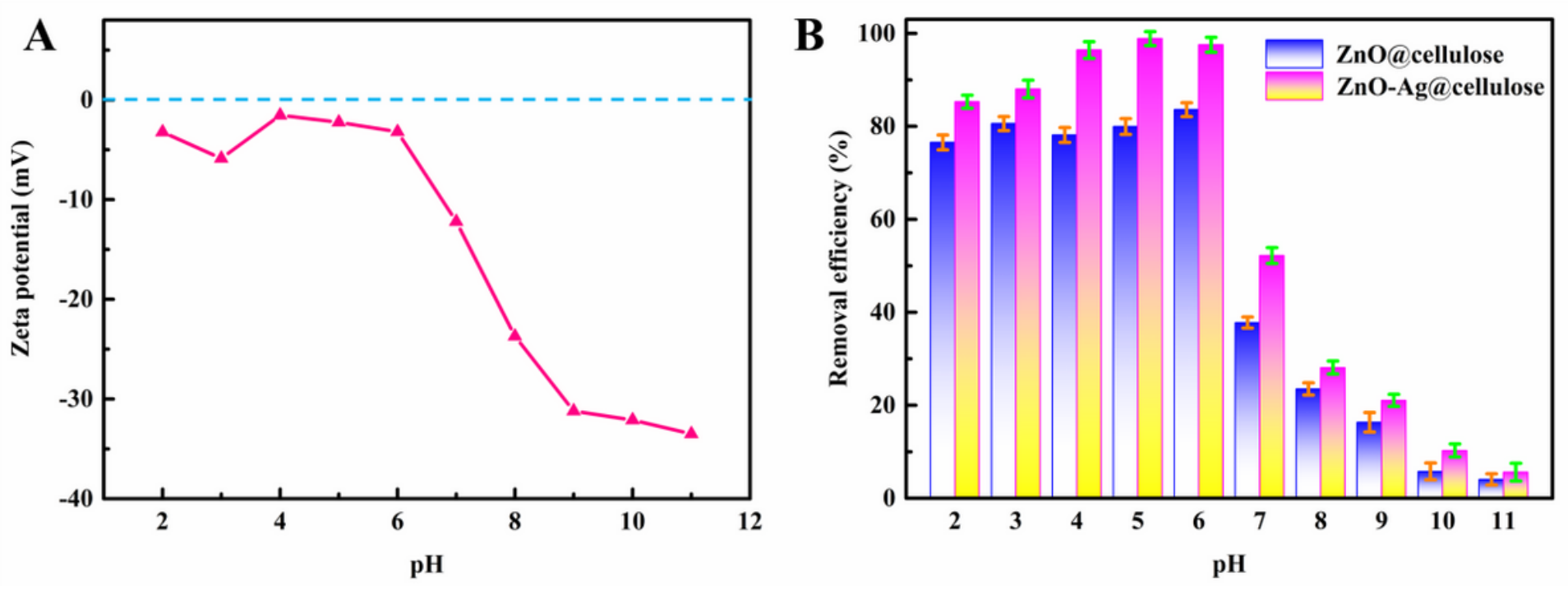

Figure 5 
Zeta potentials under various $\mathrm{pH}$ of ZAC (A); Effect of $\mathrm{pH}$ on the removal efficiency of Te (IV) onto ZC and ZAC (B) (Tellurium initial concentration $=40 \mathrm{mg} / \mathrm{L}$, adsorbent dosage $=30 \mathrm{mg}$, temperature $=25^{\circ} \mathrm{C}, \mathrm{pH}$ adjusted with $0.1 \mathrm{~mol} / \mathrm{L} \mathrm{NaOH}$ and $\mathrm{HCl}$ ).
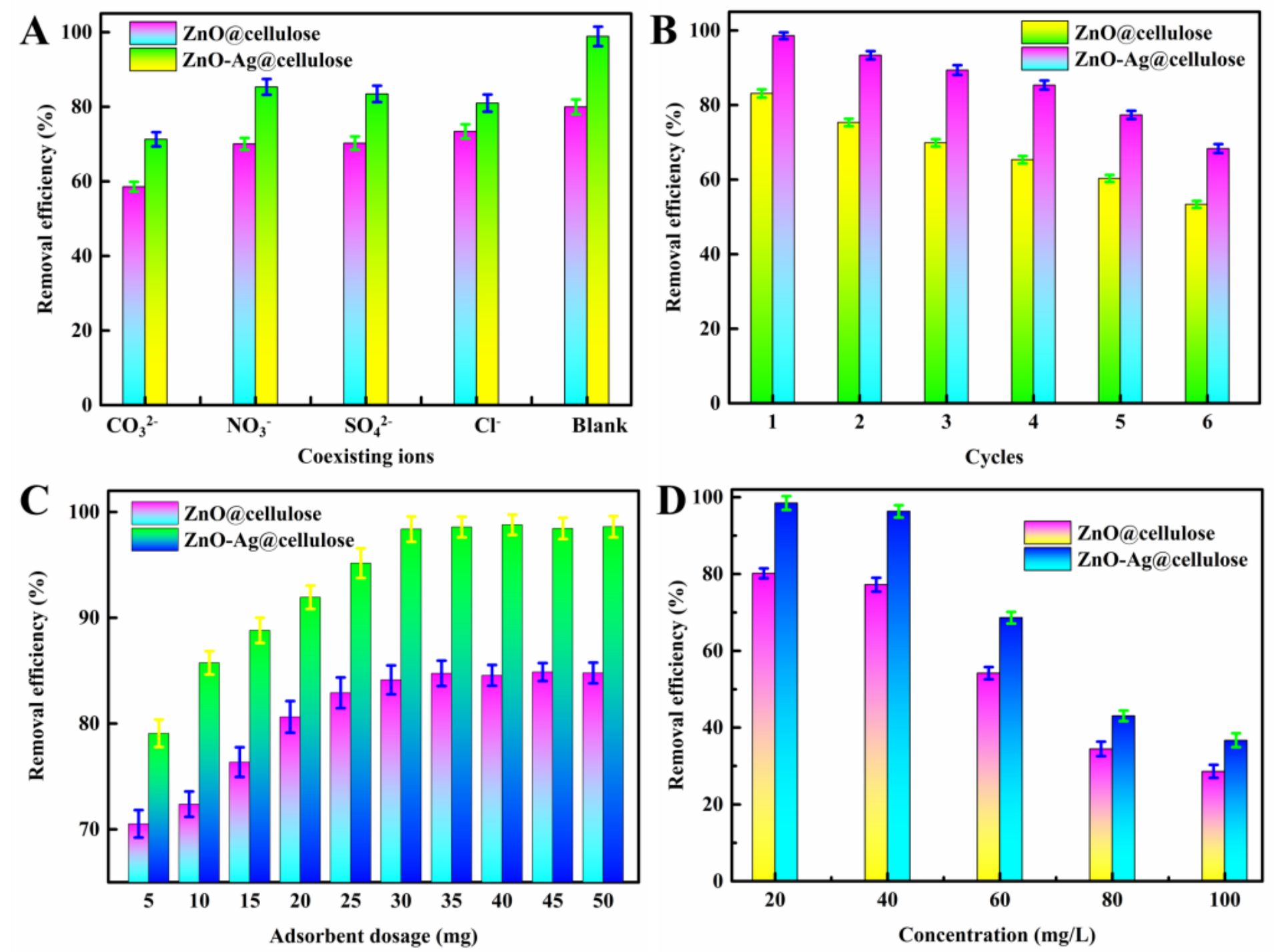

Figure 6

Effect of coexisting anions on removal efficiency of ZC and ZAC (A); removal rate of tellurium by ZC and ZAC at different regeneration cycles (B); effect of different adsorbent dosage on removal efficiency of ZC and ZAC (C); different initial concentrations of tellurium adsorption results (D). (Tellurium initial concentration $=40 \mathrm{mg} / \mathrm{L}$, adsorbent dosage $=30 \mathrm{mg}$, temperature $=25^{\circ} \mathrm{C}, \mathrm{pH}=5$.) 


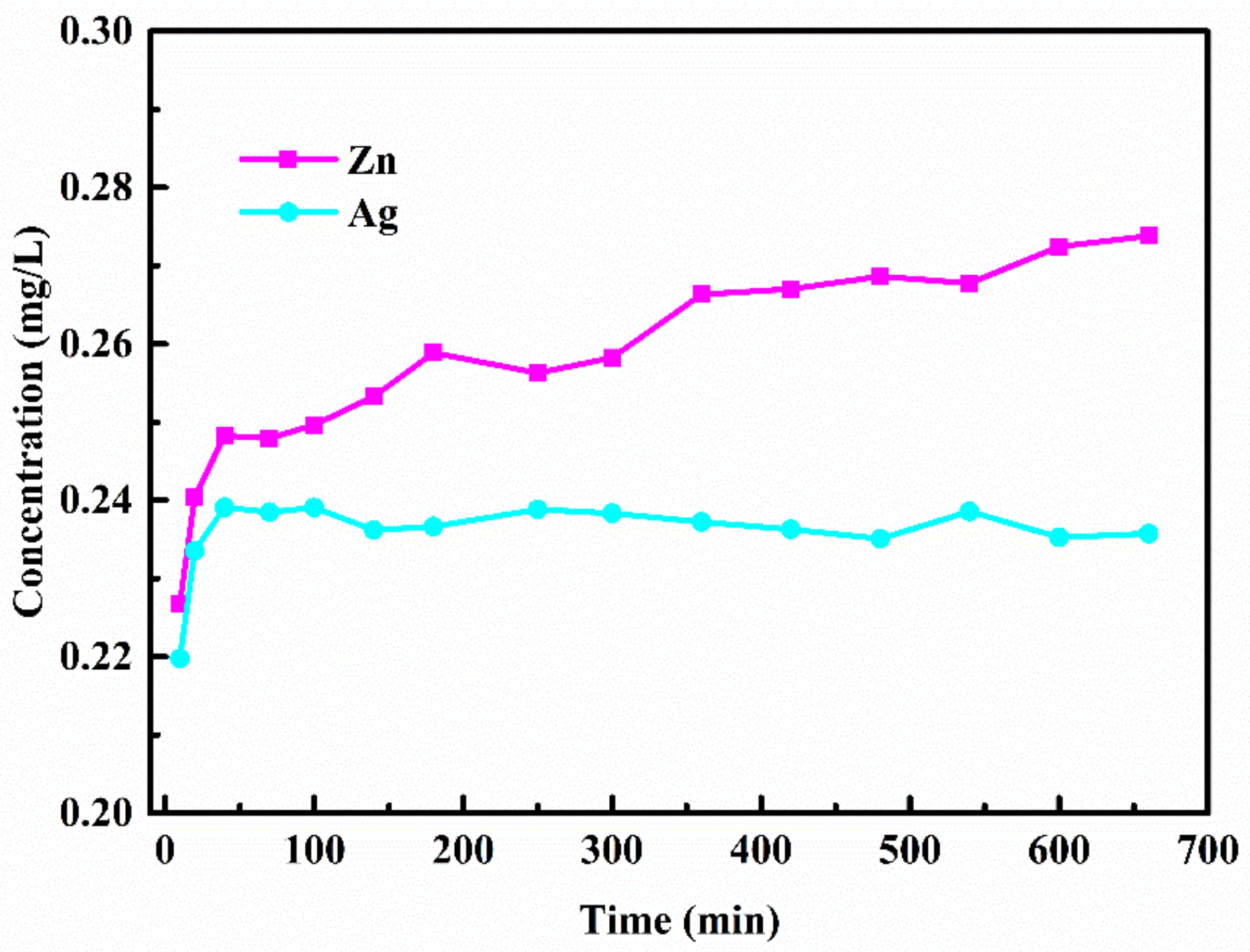

Figure 7

The concentration of $\mathrm{Zn}$ and $\mathrm{Ag}$ changes with time increase. 


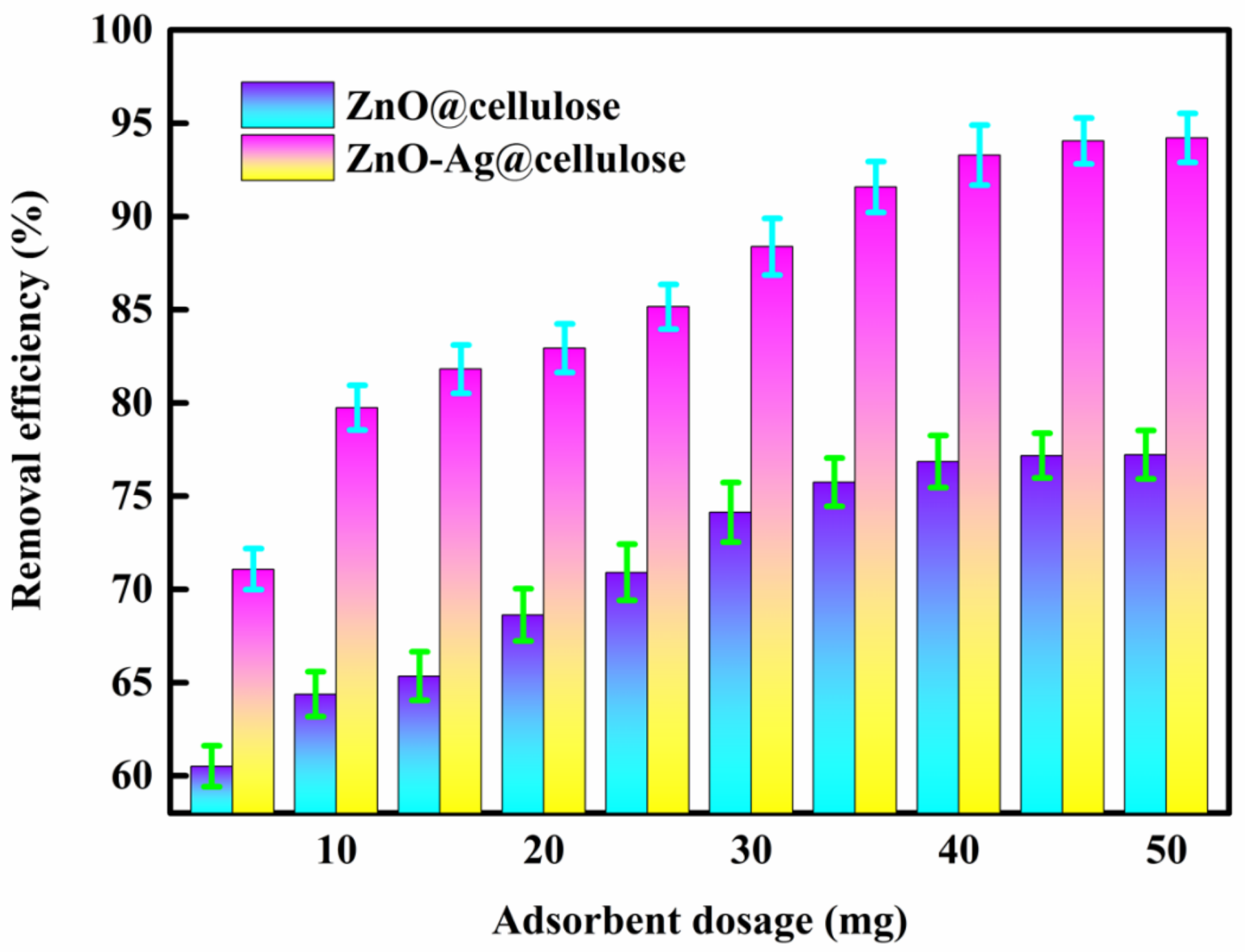

Figure 8

Antifouling performances of ZC and ZAC under different adsorbent dosage. 

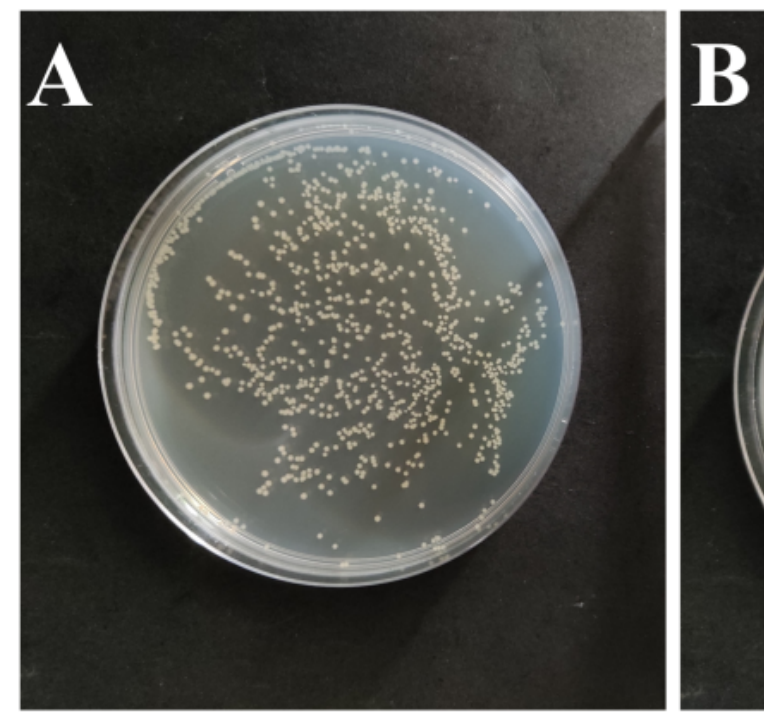

D

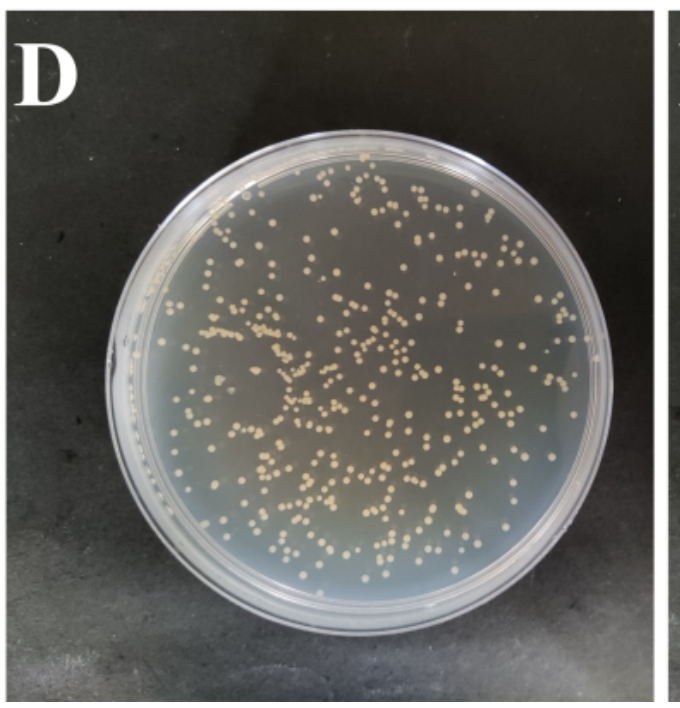

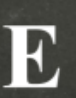

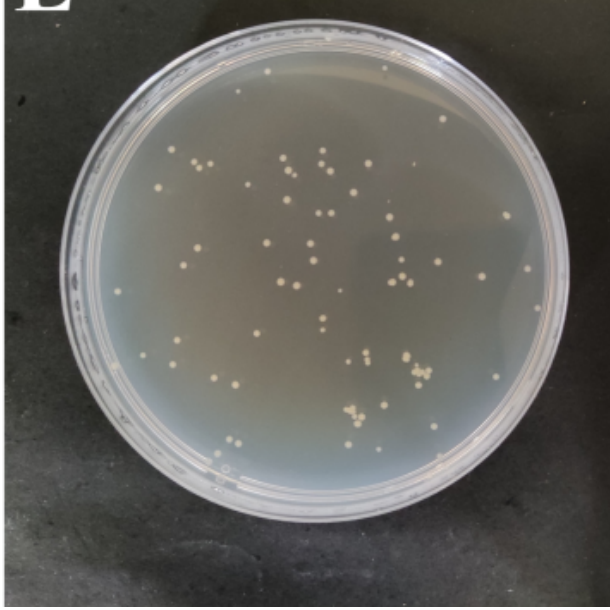

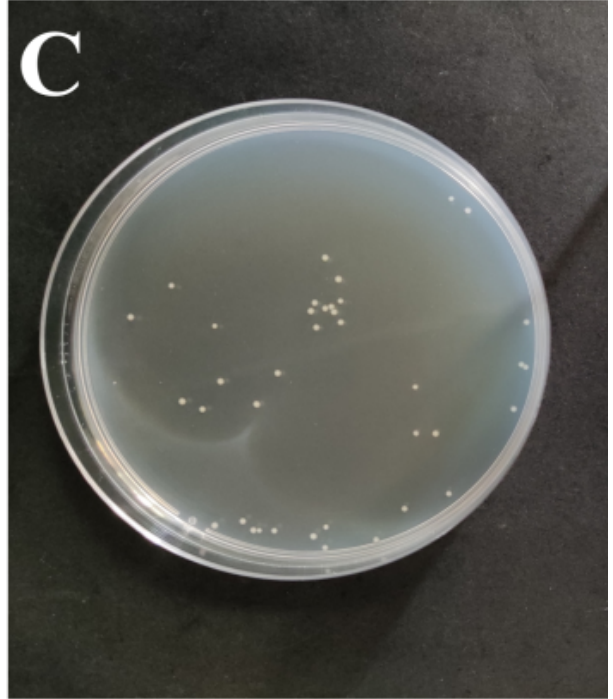
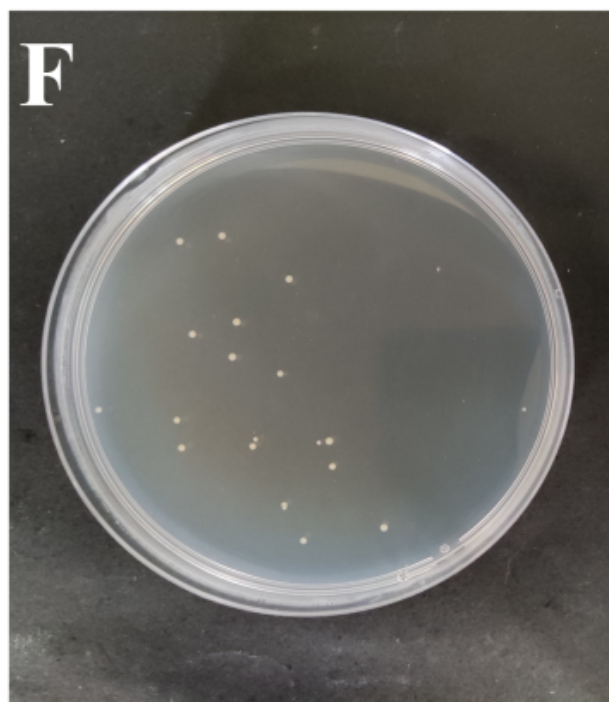

\section{Figure 9}

Antibacterial properties of adsorbent against E. coli: (A) control, (B) ZC, (C) ZAC, Antibacterial properties of adsorbent against S. aureus: (D) control, (E) ZC, (F) ZAC.

\section{Supplementary Files}

This is a list of supplementary files associated with this preprint. Click to download.

- Scheme1.png

- Scheme2.png

- SupportingInformation.docx 